\title{
Efecto de la suplementación con forrajes arbustivos sobre el desempeño productivo de conejos (Oryctolagus cuniculus)
}

\author{
Verónica Marcela Calderón Bedoya ${ }^{1}$, Raúl Velásquez Vélez ${ }^{2}$ y Miguel Angel Castaño Benitez ${ }^{3}$. \\ 1Especialista en Agronegocios, Docente Politécnico Colombiano Jaime Isaza Cadavid \\ veronica_calderon86103@elpoli.edu.co \\ 2Doctor en Ciencias Animales, Docente Politécnico Colombiano Jaime Isaza Cadavid. ravelasquez@elpoli.edu.co \\ ${ }^{3}$ Estudiante Ingeniería Agropecuaria, Politécnico Colombiano Jaime Isaza Cadavid \\ miguel_castano86181@elpoli.edu.co
}

\section{RESUMEN}

Actualmente existe una alta demanda de alimentos balanceados destinados a la ceba de animales que resultan costosos, por lo tanto, los alimentos balanceados no convencionales son una alternativa para obtener buenos resultados a bajo costo; planteando como objetivo, evaluar el efecto de la suplementación de diferentes forrajes en el desempeño productivo y rendimiento de la canal de 24 conejos machos raza Nueva Zelanda, distribuidos en 4 tratamientos con 6 individuos cada uno, determinando ganancia de peso, rendimiento de canal y costos de alimentación. Se realizó un análisis de medidas repetidas en el tiempo con el paquete estadístico SAS, encontrando que no hay diferencias significativas $(p \leq 0,05)$ entre el Botón de oro y el alimento comercial, con respecto rendimiento de la canal, identificando una disminución en costos, concluyendo que la suplementación con materias primas no convencionales es una alternativa para reducir los costos de producción, hasta un $21,3 \%$.

Palabras claves: Seguridad alimentaria, alimentación, costos, aumento de peso, forrajes.

Recibido: 05 de abril de 2021. Aceptado: 20 de julio de 2021

Received: April 05, 2021. Accepted: July 20, 2021

\section{EFFECT OF THE SUPPLEMENTATION WITH BUSH FORAGES ON THE PRODUCTIVE PERFORMANCE OF RABBITS (Oryctolagus cuniculus)}

\begin{abstract}
Currently there is a high demand for balanced feeds intended for fattening animals that are expensive, therefore, unconventional balanced feeds are an alternative to obtain good results at low cost, consequently, the objective was to evaluate the effect of supplementation of different forages in the productive and carcass performance of 24 male New Zealand breed rabbits, distributed in 4 treatments with 6 individuals each one, measuring weight gain, carcass weight and feeding costs. It performed a repeated measures analysis over time with the SAS statistical package, there are no significant differences $(p \leq 0,05)$ between Mexican sunflower and commercial feed, with respect to carcass performance, identifying a decrease in costs, concluding that supplementation with unconventional raw materials is an alternative to reduce production costs, up to $21,3 \%$.
\end{abstract}

Keywords: Food safety, feeding, costs, weight gain, forages.

Cómo citar este artículo: V. Calderón, R. Velásquez, M. Castaño. "Efecto de la suplementación con forrajes arbustivos sobre el desempeño productivo de conejos (Oryctolagus cuniculus)", Revista Politécnica, vol.17, no.34 pp.30-38, 2021. DOI: https://doi.org/10.33571/rpolitec.v17n34a2 


\section{INTRODUCCIÓN}

La producción cunícola a nivel global genera alrededor de 925 millones de cabezas de conejos al año equivaliendo a 883.936 toneladas de carne, según la FAO [1] destacándose Asia como uno de los principales productores con un $56,7 \%$ de la industria mundial, seguido por Sudamérica, $30 \%$ donde Colombia se caracteriza por ser el país con mayor crecimiento anual $(15,2 \%)$, el cual genera más de mil millones de toneladas, siendo 37.800 unidades productivas las que año tras año crían 735.000 conejos según el DANE [2] . Aunque el consumo a nivel interno se encuentra en una fase inicial, la cría de conejos se considera una actividad ideal para la soberanía alimentaria de las familias campesinas, ya que proveen alimento de alto valor biológico como proteína animal a partir del consumo de una amplia variedad de forrajes [3].

Entre las principales ventajas de la producción cunícola, se encuentran: el corto intervalo generacional, la alta prolificidad y el provechoso rendimiento de la carne [1]. Pero, la alimentación de estos animales a nivel industrial presenta diferentes limitaciones, entre las cuales, cabe resaltar el alto costo del alimento balanceado que se elabora con base en maíz y soya, los cuales son importados [3; 4], esto incentiva la utilización de otras materias primas no convencionales que tienen una amplia distribución, por lo tanto, son alternativas para una alimentación balanceada elaborada en finca, que posibilitan obtener una mayor rentabilidad para el campesino [4], como es el caso de las forrajeras arbóreas o arbustivas [5; 6].

La harina de Botón de Oro (Tithonia diversifolia), se ha utilizado en las dietas para conejos en crecimiento sin alterar el consumo, ni la salud de los mismos [7], también, el silo, ha sido útil como complemento alimenticio de ovinos [8], conteniendo hasta un $27,4 \%$ de proteína bruta (PB) [9]. El Quiebrabarrigo (Trichanthera gigantea) puede ofrecerse a conejos, cuyes, rumiantes, equinos, cerdos, gallinas, pollos, patos, gansos y pavos, posee un $16,94 \%$ de proteína cruda [10], mientras que el Ramio (Boehmeria nivea) es una fuente común de proteína para monogástricos (26,43\% PB) [12], tales como el conejo, el cerdo y la gallina.

Tabla 1. Bromatológico materias primas utilizadas.

\begin{tabular}{cccccc}
\hline $\begin{array}{c}\text { MATERIA } \\
\text { PRIMA }\end{array}$ & MS $(\%)$ & $\mathrm{PB}(\%)$ & $\mathrm{FDN}(\%)$ & $\mathrm{FDA}(\%)$ & FUENTE \\
\hline $\begin{array}{c}\text { TITHONIA } \\
\text { DIVERSIFOLIA }\end{array}$ & 18,21 & 28,49 & 40,44 & 24,11 & {$[9]$} \\
$\begin{array}{c}\text { TRICHANTHER } \\
\text { A GIGANTEA }\end{array}$ & 12,78 & 16,94 & 35,7 & 16,8 & {$[10][11]$} \\
$\begin{array}{c}\text { BOEHMERIA } \\
\text { NIVEA }\end{array}$ & 18,2 & 24,4 & 48,5 & 45,4 & {$[12]$} \\
$\begin{array}{c}\text { HARINA DE } \\
\text { ZEA MAYS }\end{array}$ & 87,67 & 8,71 & 60,1 & 35,7 & {$[13][14]$} \\
$\begin{array}{c}\text { HARINA DE } \\
\text { GLYCINE MAX }\end{array}$ & 79,4 & 39,9 & - & - & {$[15]$} \\
\hline
\end{tabular}

\section{MATERIALES Y MÉTODOS}

\subsection{Ubicación}

El estudio se realizó en la granja Román Gómez Gómez del Politécnico Colombiano Jaime Isaza Cadavid, ubicada en el municipio de Marinilla (Antioquia), en la vereda Primavera, localizada a $2150 \mathrm{msnm}$, con una temperatura media de $17,5^{\circ} \mathrm{C}$, correspondiente a una zona de vida Bosque húmedo montano bajo (Bh-MB).

\subsection{Animales y manejo.}


Se utilizaron 24 machos de la raza Nueva Zelanda, con un peso promedio $1222,7 \pm 114,5 \mathrm{~g}$ y una edad de 45 días. Se formaron 4 grupos de 6 animales cada uno y permanecieron en confinamiento completo en jaulas de $35 \times 50 \mathrm{~cm}$ cada grupo. Cada jaula tenía un comedero doble y bebederos automáticos. Se alimentaban con forraje de Quiebrabarrigo (Trichanthera gigantea) fresco a razón de $250 \mathrm{~g}$ por jaula y se suplementaron con el alimento balanceado $(A B)$ según el tratamiento, con repeticiones de 2 veces en el día. Se realizaron los cortes de Botón de Oro (Tithonia diversifolia), Ramio (Boehmeria nivea) y Quiebrabarrigo (Trichanthera gigantea); luego, se deshidrataron y reducían el tamaño de las partículas con una picapastos.

Considerando estas dietas como balances isoproteicos se implementó la técnica de Pearson modificado; igualando el $18 \%$ que posee el alimento comercial con el contenido proteico del material vegetal a evaluar, estando las mezclas compuestas de la siguiente manera:

El AB comercial se utilizó como tratamiento testigo, teniendo como orden de tratamientos, los siguientes:

Tratamiento 1 (T1): Control, AB comercial.

Tratamiento 2 (T2): AB basado en Botón de oro (Tithonia diversifolia) $(14,5 \%)+$ Harina de maíz (Zea mayz) $(24,2 \%)+$ Soja (Glycine max) $(61,4 \%)$

Tratamiento 3 (T3): AB basado en Ramio (Boehmeria nívea) (18,9\%) + Harina de maíz (Zea mayz) (22,8\%) + Soja (Glycine max) $(58,3 \%)$

Tratamiento 4 (T4): AB basado en Quiebrabarrigo (Trichanthera gigante) $(1,6 \%)$ + Harina de maíz (Zea mayz) $(27,7 \%)+$ Soja (Glycine max) $(70,7 \%)$.

El tiempo de ceba estuvo comprendido por 5,7 semanas, con 10 días de adaptación a la dieta, donde todos los animales fueron seleccionados con condiciones similares de acuerdo al peso vivo y sexo. Suministrando el alimento en comederos dobles y el agua en bebederos automáticos, ambos fueron a voluntad de consumo, además, en horas de la mañana recibieron $250 \mathrm{~g} /$ día de Quiebrabarrigo recién cortado como alimento base para cada jaula. (83,3 g/animal).

Para los animales testeados se comienza con un peso inicial similar, oscilando entre 1393,3 - $1149 \mathrm{~g}$, llevando a cabo el beneficio de los animales a las 12 horas de ayuno, tomando el peso vivo y de canal como referencia para determinar rendimiento de la canal.

\subsection{Diseño estadístico}

Se implementó un diseño completamente aleatorizado, con 24 animales sometidos a 4 tratamientos, en unidades experimentales con grupos de 6 animales, utilizando el paquete estadístico SAS, se realizó el análisis estadístico evaluando, peso final, ganancia de peso total, ganancia de peso diario, rendimiento de la canal y conversión alimenticia por tratamiento.

El peso inicial de los animales $(\mathrm{PI})$ se consideró covariable, para corregir, ya que es significativamente diferente en los tratamientos.

\section{RESULTADOS}

Los datos de peso inicial (PI), peso final (PF), ganancia de peso total (GPT), ganancia de peso diario (GPD), rendimiento de la canal (\%RDTO), conversión alimenticia (CONV) por tratamiento están descritos en la Tabla 2.

Tabla 2. Comparaciones entre los suplementos alimenticios

\begin{tabular}{ccccc}
\hline & T1 & T2 & T3 & T4 \\
\hline PI (G) & $1393,3 \mathrm{~A}^{*}$ & $1182 \mathrm{~B}$ & $1149,3 \mathrm{~B}$ & $1166 \mathrm{~B}$
\end{tabular}




\begin{tabular}{|c|c|c|c|c|}
\hline PF (G) & $2810 \mathrm{~A}$ & 1971B & 1735,8 в & $1558 \mathrm{~B}$ \\
\hline GPT (G) & 1416,7 A & 789 в & $586,5 \mathrm{BC}$ & $392 \mathrm{C}$ \\
\hline GPD (G) & $35,4 \mathrm{~A}$ & 19,7 в & 14,7 вС & $9,8 \mathrm{C}$ \\
\hline RDTO (\%) & $48,8 \mathrm{~A}$ & $46,1 \mathrm{~A}$ & $46,9 \mathrm{~A}$ & $47,4 \mathrm{~A}$ \\
\hline $\begin{array}{c}\mathrm{CONV} \text { (KG } \\
\mathrm{MS} / \mathrm{KG} \\
\mathrm{GAN})\end{array}$ & $3,4 \mathrm{C}$ & $6,10 \mathrm{~B}$ & $8,18 \mathrm{~B}$ & $12,26 \mathrm{~A}$ \\
\hline
\end{tabular}

AB comercial ( $T 1$ ), AB basado en Botón de oro (T2), AB basado en Ramio (T3) y AB basado en Quiebrabarrigo (T4).

Los pesos iniciales $(\mathrm{PI})$ de los animales fueron muy similares, mayores a $1000 \mathrm{~g}$, con ganancias de peso totales (GPT) elevadas en el AB comercial $(1416 \mathrm{~g}$ ) debido a que presenta condiciones favorables considerándose una dieta ideal para la producción cunícola por su alto contenido de carbohidratos solubles, siendo los tratamientos en base a Botón de oro (T2) y Ramio (T3), los que reflejan ganancias de peso y conversiones alimenticias similares, por debajo del AB comercial por el contenido de carbohidratos estructurales (\%FDN) reflejado en la tabla 1 , contrario al tratamiento $\mathrm{T} 4$, el cual presenta una ganancias de peso paulatinas y poco eficientes durante las 5,7 semanas de engorde.

Debido a que el Botón de oro (Tithonia diversifolia) posee altos niveles de nutrientes digestibles para conejos de engorde en crecimiento, siendo una alternativa alimenticia en condiciones tropicales; en un estudio posterior Nieves et al. [18] encontraron que favoreció disminuir el uso del AB comercial (T1) además los animales obtuvieron adecuadas ganancias de peso debido a su alta aceptación, además cuenta con una propagación y manejo del cultivo eficientes logrando reducir los costos de producción [19].

Pérez et al. [16] aseguran que el Ramio (Boehmeria nívea) es una buena alternativa alimenticia basados en su valor nutritivo, ganancia de peso y conversión alimenticia, en el presente estudio fue el tratamiento que arrojó una ganancia diaria de peso similar al Botón de oro, pero fue el tratamiento con mayores pérdidas de alimento, sin embargo, [19] mencionan que el Ramio posee alta palatabilidad lo cual induce un mayor consumo del mismo, esta es la razón por la cual se considera un alimento base para la cunicultura, pero es recomendable suministrarlo en compañía del $A B$ para obtener un efecto positivo porque se incrementa la asimilación de los nutrientes del Ramio, en especial de materia seca y proteína.

Por otra parte, Nieves et al. [6] evaluaron diferentes dietas elaboradas con materias primas no convencionales y la suplementación con Quiebrabarrigo, concluyendo que el consumo del alimento fue similar al comercial pero hubo una menor ganancia diaria de peso para las formulaciones no convencionales, lo cual se comprueba con estos resultados, sin embargo, con el Quiebrabarrigo se obtuvo un peso final similar al logrado con el ramio pero entre los tratamientos fue el suplemento que arrojó menor ganancia diaria de peso, aunque Arango y Quintero [20] afirman que con la inclusión hasta un 30\% de ramio en la dieta de conejos de ceba se logran buenos resultados a bajo costo. 


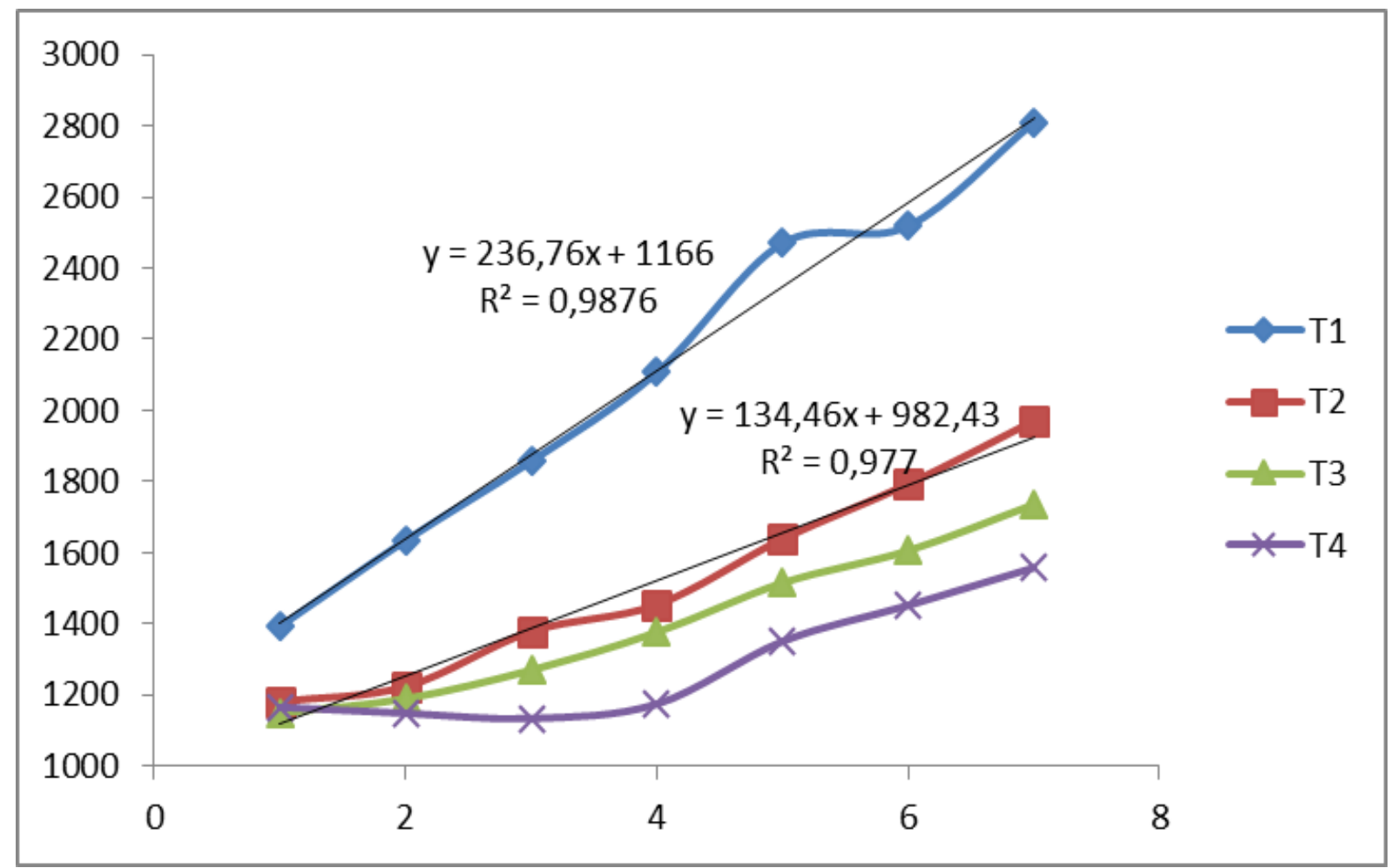

Figura 1. Curva de crecimiento de los animales de cada tratamiento en el tiempo

El comportamiento de aumento de peso, en el cual sobresale el $A B$ comercial presentando ganancias de peso en menor tiempo, los tratamientos con base a forrajeras se comportan de manera positiva, pero lentamente, se evidencia la eficiencia en el engorde con $A B$ comercial, pero al tiempo se puede observar como el T2 presenta un aumento muy por encima de los otros dos tratamientos, mostrándose como la alternativa más eficiente, excluyendo al tratamiento control.

Tabla 3. Costos de alimentación $(\$ C O P)$

\begin{tabular}{ccccc}
\hline & T 1 & T2 & T3 & T4 \\
\hline \$ FORRAJE & 616,1 & 616,1 & 616,1 & 616,1 \\
& & & & \\
\$ MANO DE OBRA & 306,9 & 306,9 & 306,9 & 306,9 \\
\$ AB & & & & \\
TRATAMIENTO & 28.700 & 16.625 & 12.788 & 14.955 \\
\$ DIETA TOTAL & 29.623 & 17.547 & 13.711 & 15.877 \\
\$ DIETA X & 4.937 & 2.925 & 2.285 & 2.646 \\
ANIMAL & 3.485 & 2.743 & 2.945 & 5.332 \\
\$ KG PV & 0 & $21,3 \%$ & $15,5 \%$ & $53 \%$ \\
\% AHORRO & & & &
\end{tabular}


La alimentación basada en forrajes ricos en proteína, Botón de oro $(24,13 \%)$, Ramio $(26,43 \%)$ y Quiebrabarrigo (18,6\%), entran a competir significativamente con el $A B$ comercial (18\%), siendo una alternativa idónea para el trópico donde se logran cultivar durante todo el año y obtener bajos costos de producción (Tabla 3).

El AB con Botón de Oro (T2) se caracteriza por presentar un mayor aprovechamiento debido a que el precio para la ganancia de $\mathrm{kg}$ PV es la de menor valor (2.743 COP) y presenta hasta un ahorro del $21,3 \%$ en relación con el T1. Este tipo de alimentación se puede considerar ideal para el engorde de conejos, en las comunidades campesinas, aunque no se tiene las mismas ganancias de peso en tan poco tiempo como se observa en el tratamiento control, si se pueden llegar a engordar los conejos con un poco más de tiempo, disminuyendo el valor, logrando cultivar los forrajes.

Realizando estas alimentaciones para herbívoros, se encuentran características anatómicas notorias como lo son, los fermentadores cecales como son los conejos, los cuales ingieren las primeras heces blandas en horas de la mañana (cecotróficos) [21], los cuales realizan el aprovechamiento máximo de los nutrientes encontrados en los forrajes consumidos, es por eso que se hace uso de plantas forrajeras con características similares, tanto en aporte nutricional, como en precio en el mercado, de igual manera se observa que poseen precios semejantes en el mercado, determinando una marcada diferencia en los consumos totales y por ende en los desperdicios que se generan en los comederos, expresados anteriormente en la Tabla 3.

\section{CONCLUSIONES}

En la alimentación tradicional usando alimentos balanceados comerciales, se obtiene ganancias de pesos en menor tiempo, pero a un costo mayor, que si se hace uso de material vegetal con características nutricionales propicias para herbívoros como los conejos, los cuales aprovechan al máximo los nutrientes encontrados en los forrajes suministrados, dando como resultado una alternativa de alimentación a menor costo $(21,3 \%)$ para los productores, que aunque se puede disminuir las ganancias de peso y peso final en el periodo de tiempo evaluado, seguirá siendo más económico una alimentación alternativa con forraje, especialmente Botón de oro (Tithonia diversifolia), que una alimentación convencional.

Basándose en el peso final de los animales y su precio de venta $(\$ 12.000 / \mathrm{kg})$ el tratamiento T2 presentó resultados favorables al compararlo con el alimento comercial.

Al incentivar la alimentación tradicional se puede trabajar con material vegetal de fácil adquisición, mejorando de esta manera los costos de producción del alimento y aumentando la mano de obra de la región, donde se instalen estos tipos de cultivos destinados para la nutrición animal.

\section{AGRADECIMIENTOS}

Agradecemos al semillero del Grupo de Investigación en Biotecnología Animal (GIBA) por acogernos y brindarnos la motivación a través de nuestros docentes por la investigación y a la coordinación de granjas que promueven los espacios de la institución con fines investigativos.

\section{REFERENCIAS}

[1] FAO. (2019). FAOSTAT. http://www.fao.org/faostat/es/\#data/QL 
[2] Ministerio de Agricultura y Desarrollo Rural, Colombia. (2018, Mayo). MinAgricultura busca apoyar a cerca de 39 mil familias productoras de carne de conejo, conformando la cadena productiva. https://www.minagricultura.gov.co/noticias/Paginas/MinAgricultura-busca-apoyar-acerca-de-39-mil-familias-productoras-de-carne-de-conejo,-conformando-la-cadenaproductiva.aspx

[3] Clavijo, L. M. (2018). Análisis de viabilidad técnica y de mercado para la implementación de un sistema productivo y de comercialización de carne de conejo en el municipio de San Agustín (Huila). [Tesis de pregrado, Universidad Nacional Abierta y a Distancia UNAD]. Repositorio Institucional UNAD. https://repository.unad.edu.co/handle/10596/20938

[4] Martínez, R., Santos, R., Ramírez, L., y Sarmiento, L. (2010) Utilización de Ramón (Brosimum alicastrum Sw.) y Cayena (Hibiscus rosasinensis L.) en la alimentación de conejos. Zootecnia Tropical, 28(2), 153-161. http://www.scielo.org.ve/scielo.php?pid=S0798$72692010000200002 \&$ script=sci_arttext

[5] Palma, O., y Hurtado, E. (2010) Comportamiento productivo de conejos durante el período de crecimiento-engorde alimentados con frutos de mango (Mangifera indica) en sustitución parcial del alimento balanceado comercial. IDESIA 28(1), 33-37. http://www.scielo.cl/scielo.php?script=sci_arttext\&pid=S0718-34292010000100005

[6] Nieves, D., López, D., y Cadena, D. (2001). Alimentación de conejos de engorde con dietas basadas en materias primas no convencionales y suplementación con Tríchanthera gigantea. Revista Unellez de Ciencia y Tecnología. Volumen especial, 60-66. http://www.saber.ula.ve/revistaunellez/pdfs/60-66.pdf

[7] Sarwatt, S., Laswi, G., y Ubwe, R. (2003) Evaluation of the potencial of Trichanthera gigantea as a source of nutrients for rabbits diets under small-holder production system in Tanzania. Livestock Research for Rural Development 15(11), 110-114. http://www.lrrd.org/lrrd15/11/sarw1511.htm

[8] Martínez, M., Motta, W., Cervera, C., y Pla, M. (2005) Feeding mulberry leaves to fattening rabbits: effects on growth, carcass characteristics and meat quality. Animal Science 80(3), 275281. https://doi.org/10.1079/ASC41110275

[9] Verdecia, D. M., Ramírez, J. L., Leonard, I., Álvarez, Y., Bazán, Y., Bodas, R., Andrés, S., Álvarez, J., Giráldez, F., y López, S. (2011). Calidad de la Tithonia diversifolia en una zona del Valle del Cauto. Revista Electrónica de Veterinaria REDVET, 12(5), 1-13. https://www.redalyc.org/articulo.oa?id=63622168004.

[10] Sossa, L. S.; Higuita E., P.; Guerra, C. L. y Mosquera, J. J. (2020). Inclusión de harina de Trichanthera gigantea y Morus alba en la alimentación de pollos de engorde. Revista Universidad Católica de Oriente, 31(46), 167-180. http://200.9.158.34/index.php/uco/article/view/324/402 
[11] Ospina, S., Rosales, M., y Ararat, J. E. (2002). Variación genotípica en la composición química y digestibilidad de Trichanthera gigantea. Agroforestería de las Américas, 9(33), 24-32. http://hdl.handle.net/11554/6918

[12] Naranjo, J. F. y Cuartas, C.A. (2011). Caracterización nutricional y de la cinética de degradación ruminal de algunos de los recursos forrajeros con potencial para la suplementación de rumiante en el trópico alto de Colombia. Revista CES Medicina Veterinaria y Zootecnia, 6(1), 919. https://www.redalyc.org/articulo.oa?id=321428105002

[13] Vivas, W. A. C. (2018). Fracciones químicas del maiz (Zea maíz) sometido a procesos sucesivos de ensilaje y amonificación utilizado en la alimentación de bovinos en tame arauca. Agricolae \& Habitat, 1(1), 1-26. https://doi.org/10.22490/26653176.2340

[14] Villamagua, L. M. (2013). Elaboración de una mezcla alimenticia a base de chocho y maíz, que contribuya a mejorar el estado nutricional de los niños y niñas menores de 5 años de los barrios San Vicente, La Loma, Sagrado Corazón, Cochaloma, San Vicente, de la Comunidad de Cangahua. [Tesis pregrado, Pontificia Universidad Católica del Ecuador]. Repositorio institucional PUCE. http://repositorio.puce.edu.ec/handle/22000/7517

[15] Espejo, C. (2004). DETERMINACIÓN DEL VALOR NUTRICIONAL DE LA SOYA INTEGRAL (Glycine max) EN LA ALIMENTACIÓN DE LA TILAPIA ROJA (Oreochromis sp.). Revista Electrónica de Ingeniería en Producción Acuícola. 1(1). https://revistas.udenar.edu.co/index.php/reipa/article/view/1494

[16] Moreno F.O., y Molina, D. (2007). Buenas prácticas agropecuarias [BPA] en la producción de ganado de doble propósito bajo confinamiento con caña panelera como parte de la dieta. Organización de las Naciones Unidas para la Agricultura y la Alimentación. ftp.fao.org/docrep/fao/010/a1564s/a1564s04.pdf

[17] Nieves, D., Terán, O., Cruz, L., Mena, M., y Gutiérrez, F. L. (2011). Digestibilidad de nutrientes en follaje de árnica (Tithonia diversifolia) en conejos de engorde. Tropical and Subtropical Agroecosystems, 309314. http://www.redalyc.org/articulo.oa?id=93915703030

[18] Nieves, D., Pérez, J., Jiménez, N., Calles, H., Pineda, T., y Viloria, W. (2012). Uso de follaje fresco de árnica (Tithonia diversifolia) y morera (Morus alba) en la alimentación de conejos. Revista Academia, 113-123. http://erevistas.saber.ula.ve/index.php/academia/article/view/6118

[19] González, J.C., Hahn, C.M., y Solarte, W. (2014) Características botánicas de Tithonia diversifolia (Asterales: Asteraceae) y su uso en la alimentación animal. Bol. Cient. Mus. Hist. Nat. U. de Caldas, 18(2), 45-58. http://www.scielo.org.co/scielo.php?script=sci_arttext\&pid=S012330682014000200004 
[20] Arango, J.F., y Quintero de Vallejo, V. Evaluación de tres niveles de nacedero Trichantera gigantea $(10 \%, 20 \%, 30 \%)$ en ceba de conejos. Acta agronómica, 40(3-4), 183-186. http://revistas.unal.edu.co/index.php/acta_agronomica/article/view/15496

[21] Brenes, A. (2014) Respuesta productiva de Conejos alimentados con forraje fresco de nacedero (Thithanchera gigantea, Lamiales: Acanthaceae). Cuadernos de Investigación UNED, 6(2), 205-211. http://www.redalyc.org/articulo.oa?id=515651796007 